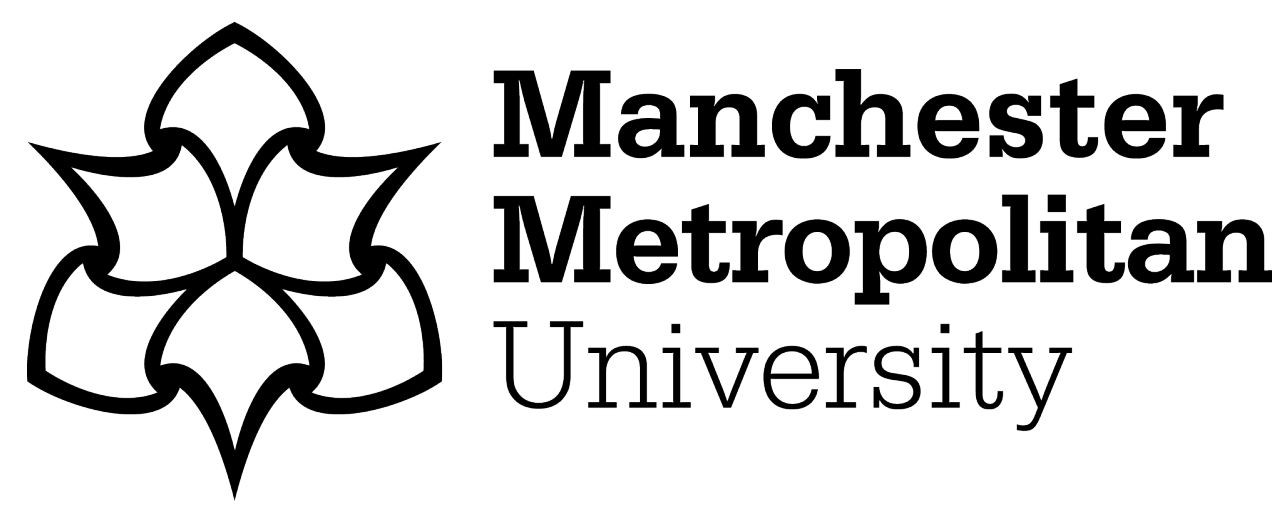

Ahsan, M, McManis, J and Hashmi, MSJ (2014) Prototype system development for wireless vehicle speed monitoring. In: 2014 9th International Symposium on Communication Systems, Networks \& Digital Signal Processing (CSNDSP), 23 July 2014 - 25 July 2014, Manchester, UK.

Downloaded from: https://e-space.mmu.ac.uk/624877/

Publisher: IEEE

DOI: https://doi.org/10.1109/csndsp.2014.6923841

Please cite the published version 


\title{
Prototype System Development for Wireless Vehicle Speed Monitoring
}

\author{
M. Ahsan*, J. McManis** and M. S. J. Hashmi* \\ *School of Mechanical and Manufacturing Engineering, Dublin City University, Dublin, Ireland \\ ${ }^{* *}$ School of Electronic Engineering, Dublin City University, Dublin, Ireland \\ md.ahsan2@mail.dcu.ie, jennifer.mcmanis@dcu.ie, saleem.hashmi@dcu.ie
}

\begin{abstract}
Vehicle speed monitoring and management of the associated data in an intelligent and efficient way is an important issue in modern transportation system in order to reduce road accidents. The aim of this work is to develop an automatic wireless system for monitoring vehicle speed on the road, identify a speeding vehicle and imposing penalty for the speeding offenders. In this work, a prototype system has been developed in a laboratory environment to generate random speed data using a mechanical wheel, measure the speed data with a Shimmer wireless sensor and transfer the data wirelessly to a client computer for further analysis. Software has been developed using a Java based socket programming technique to monitor the vehicle speed in a server computer and to send the data associated with a speeding vehicle to a remotely placed client computer. The graphical user interface (GUI) can visually display the speed of a vehicle at any particular time. The functionality of the software has been tested by simulating different traffic scenarios with low and high speed limits $(40$ and $60 \mathrm{~km} / \mathrm{hr}$ respectively). To do that a high or low speed limit can be set in the GUI. The mechanical wheel is run at different speeds and the GUI continuously displays the speed. If the vehicle speed is higher than the set speed limit for the road, the system automatically detects it and generates a report with the time of speeding, vehicle number, vehicle speed etc. to be saved in the client computer in order to take further necessary actions for the speeding offender.
\end{abstract}

Keywords-Software development; Wireless sensor; Java socket programming; Vehicle speed monitoring

\section{INTRODUCTION}

Human errors, in particular driver errors, are the causes for most of the road accidents. It is reported that over $80 \%$ of all major crashes on roads are caused from inconsiderate driving [1], e.g., vehicle over-speeding, driving after consuming alcohol, distraction during driving, non-adherence to traffic signals, tailgating, poor lane discipline etc. Managing speed data in an efficient and intelligent way is an on-going issue in modern transportation systems. Some roads have no appropriate monitoring system for identifying the speeding vehicles. Therefore, vehicles passing through these roads and exceeding the maximum speed limits can cause fatal accidents. Although many systems have been developed in order to minimise the accidents, still further work is necessary to improve the situation.

The application of a wireless sensor network (WSN) in road traffic monitoring systems can reduce accidents through wireless sensing and communication. Although there are some devices working as wireless communication systems, the operation of these systems is not in complete wireless mode. Furthermore, the existing systems are not fully automated in terms of vehicle detection, speed measurement, monitoring and data communication. Bachmann et al. [2] have reported effective speed estimation and monitoring technique for collecting vehicle data wirelessly using embedded Bluetooth - enabled devices. The system has not focused on storing data automatically. A traffic monitoring system proposed by Mastuo [3] measures the speed of moving vehicles on the road using an ultrasonic sensor. The system calculates the number of vehicles and speed from highway. The system does not expand for detecting speeding vehicle as well as report generation. Furthermore, an automated vehicle classification system using wireless accelerometers and magnetometers have been proposed by Ma et al [4] to determine speeds of the moving vehicles. The system has only considered calculating the speeds of moving vehicles.

The existing systems focus on detecting vehicle and calculating speed rather than developing a facility for displaying current status of the vehicle speed and increasing consciousness in driver's mind to control speed on the road. Systems are also unavailable to manage and store speeding data automatically in a central database, which eliminates any need for human involvement.

This research proposes a wireless sensor based framework for vehicle speed monitoring and controlling [5]. In general, a sensor (accelerometer-based) and other auxiliary devices are used to detect a vehicle moving on the road. The vehicle speed is calculated by the system and cross checked with a speed limit set for a particular road. If the detected speed is greater than the speed limit, then the device sends the data to a central database wirelessly and generates a report indicating with time, road and vehicle details so that the report can be sent to the offender for implementing the speeding penalty.

The aim of the research is to develop a prototype system based on wireless sensors for detecting a vehicle, measuring and monitoring speed and managing the relevant speed data. In order to reach the aim, the objectives of this work are: (1) to develop an experimental set-up modelling vehicle motion in order to generate random speed data (2) to design and develop a wireless vehicle speed monitoring system (3) to simulate real-life traffic monitoring scenarios using the experimental set-up and monitoring system in a laboratory environment and (4) to generate reports for a speeding vehicle automatically in the system.

The organization of this article is as follows. Section II provides an overview of the relevant literature in the present context. Section III and Section IV deal with the 
developments of prototype system and software interface respectively. Section $\mathrm{V}$ demonstrates the functioning of the whole system through simulation. Finally, Section VI draws few conclusions based on the research activities.

\section{LITERATURE SURVEY}

Vehicle speed monitoring is one of the crucial components of Intelligent Transportation System (ITS). Conventional systems include a number of techniques for data acquisition from speeding vehicles [6]. In one of such traffic monitoring system [7] data related to traffic is collated and sent using a short messaging system via a wireless network. In modern times, online traffic monitoring system is an electronic system that collects data over time relating to a location with built-in sensors. The electronic online traffic monitoring has replaced chart recorders in different applications [8].

The literature reports a number of programming interfaces with existing traffic monitoring devices. Zhou et al. [9] used principal component analysis in the programming interface. In CarTel network [10], the moving vehicles collected data, and log them to their local intermittently connected database. Once the wireless network connectivity is available, the collected data is transmitted to the portal via the software. The portal is available to the users for further monitoring of the moving vehicles. The information collected from the speeding vehicles by the traffic monitoring system is managed by the base stations in numerous ways. Floating data contains information on time and location provided by positioning systems and the information collected from various vehicle sensors [11].

The use of a traffic monitoring system based on mobile sensor networks coupled with programming interface has proven helpful in determining the speeds of moving vehicles on the road. The speeds of the moving vehicles are computed and at the same time, the performance of this technique is monitored using two traffic status estimation algorithms [12].

'TrafficView' [13] is a wireless traffic monitoring system with programming interface that provides the driver with a dynamic view of the road traffic. The 'TrafficView' software on the node of the network periodically updates the speed status of the vehicle.

'TrafficSense' uses smartphones to establish an idea where a microphone, camera, GPS, accelerometer can be used with phones as traffic sensing function [14]. The system is able to detect the audio data and send it to a base station for further processing. It sends the collected data to a server. Computing, communication and sensing features are present in this system. TrafficSense uses the accelerometer on a phone to sense road and traffic conditions.

'Traffic-Dot', using wireless sensor networks has been reported by Coleri et al. [15]. The monitoring arrangement enables the wireless sensor network, particularly at the sensor nodes, to collect traffic information for measuring the vehicles' speeds with a node pair. The collected information is then forwarded to the access point over radio. The components of the 'Traffic-Dot' system are a built-in processor, a radio, a magnetometer, a battery, and a cover for protecting the system from the moving vehicles. The magnetic sensor data estimates the speeds of the moving vehicles.
Karthikeyan et al. [16] report how a GPS transceiver, receiver can collect data from the moving vehicles on highway and compare between vehicles' speeds and the pre-set speed limit on the road. This system contains Intelligent Speed Adaptation (ISA) with a wireless transceiver. The tollgate in the highway has a transceiver that can communicate with vehicle's transceiver. A vehicle equipped with the system arrives near the tollgate, which broadcasts data. The data is saved in the vehicle module as a table of information of coordinates and speed limit. After storing the data in the memory of the system, the GPS receiver tracks its location and compares its coordinates with the received data. GPS compares between the speed limit listed in the table and speed limit from that point. The system alerts the driver by sound, displaying speed limit and indicates the local speed limit.

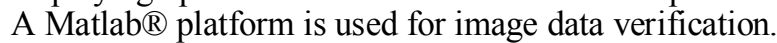

Luciani [17] has proposed a traffic monitoring system and method using one fixed transceiver located near the road that is in communication with a moving transceiver located in the vehicle running on the road. The two transceivers use Bluetooth ${ }^{\circledR}$ wireless technology. Either raw or processed data of the vehicle is sent to a Traffic Monitoring Server (TMS) using web-based technology. The system calculates the speed of the moving transceiver based on time difference. These calculations are performed at the wireless network level. The calculated speed of the moving transceiver is compared with a reference speed on the road.

Arnold et al. [18] have developed a system for managing road traffic by measuring the speed of a moving vehicle on the road with different sensor configurations. A single transducer side fire configured sensor is used in order to determine the speed of the moving vehicle. Distance measurements, phase measurements, or Doppler shift measurements are the methods used for measuring the speed of the vehicles passing through the roads.

Jorgenson et al. [19] have reported a speed-monitoring program at Indiana, USA. The collected data is managed at the speed monitoring stations, which are distributed across the highway classes based on spatial distribution, crash distribution and daily vehicle-miles travelled. Statistical models are utilised in order to locate the mean speed and compare the same with that of the daily speed distributions.

Herrera et al. [20] have assessed the feasibility of a traffic monitoring system using GPS-enabled mobile phones near Union City, California. This system performs sampling and data collection using Nokia N95 with GPS chipset thereby obtaining position data and speed of the vehicles. A prototype system architecture is implemented for testing the sampling strategies of the collected data. This system provides high accuracy in position and velocity measurements using the GPS chipsets.

A critical analysis of the available literature reveals that some of the existing road traffic monitoring systems has high equipment cost, complex installation processes, high maintenance cost and dependency on weather condition. Other systems have difficulties in managing the collected data. The difficulties at the user end of the client computers for accessing and further processing is cumbersome in some of the prevailing systems. Literature also shows that no integrated system is available for facilitating vehicle detection, speed measurement and 
monitoring, and collating and managing vehicle speed data.

\section{PRototype System DeVElopMent}

\section{A. Experimental Set-up}

An experimental set-up has been developed to generate continuous speed data, which can be measured and transferred to a computer wirelessly. The set-up includes a commercial wireless sensor (Shimmer) [21], a mechanical wheel, a tachometer coupled with a motor, a client computer and a server computer. The motor drives the wheel mounted on a shaft. The rotating wheel is considered as a moving vehicle. A speed controller controls the wheel speed and an integrated tachometer measures the wheel speed in rpm. The sensor is attached to the wheel. By setting the wheel at known speeds, a relationship between wheel speed and vibration of the wheel can be established. The sensor indirectly measures the wheel speed through sensing vibration acceleration of the wheel and communicates the speed data to the server computer wirelessly. Fig. 1 illustrates the prototype system architecture for simulating the conditions of a speeding vehicle in a laboratory environment. A wireless connection is established between the sensor and a computer through Bluetooth $^{\circledR}$. The ShimmerConnect [21] interface is configured for collecting data from the sensor in accelerometer mode. Experiments were conducted for collecting data in order to simulate single vehicle running on the road.

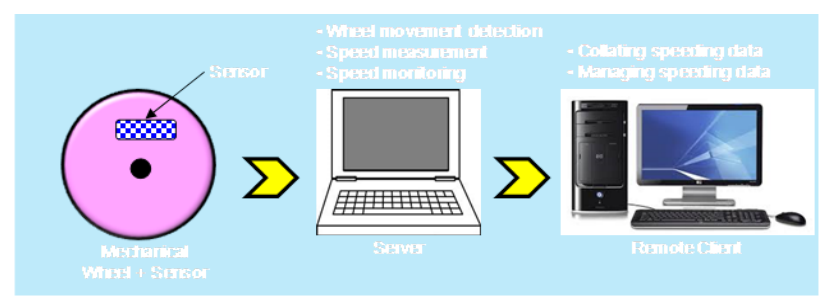

Figure 1. Prototype system architecture for monitoring vehicle speed

\section{B. Data Acquisition}

The following procedure is adopted for data acquisition from the experimental set-up and to transfer the data to the server computer:

(i) the wheel is rotated at a random speed for few minutes

(ii) average vibration acceleration is calculated for each direction in the Cartesian coordinate,

(iii) the resultant vibration acceleration is calculated from the average vibration accelerations in each direction, and

(iv) the wheel speed is calculated.

A number of principles of kinematic bodies and equations are involved in converting the average vibration acceleration into rotational wheel speed. The resultant vibration acceleration data are plotted against different wheel speeds. The resultant vibration acceleration increases proportionally with the rotational speed of the wheel. When the Shimmer sensor streams the vibration acceleration data, a real-time vibration acceleration plot is generated. This real-time plot for vibration acceleration in three Cartesian coordinates is illustrated in Fig. 2. The figure shows that with the increase of wheel speed the wavelengths in the vibration acceleration gradually decreases. This further demonstrates the evidence of the linear relationship between the acceleration and wheel speed.

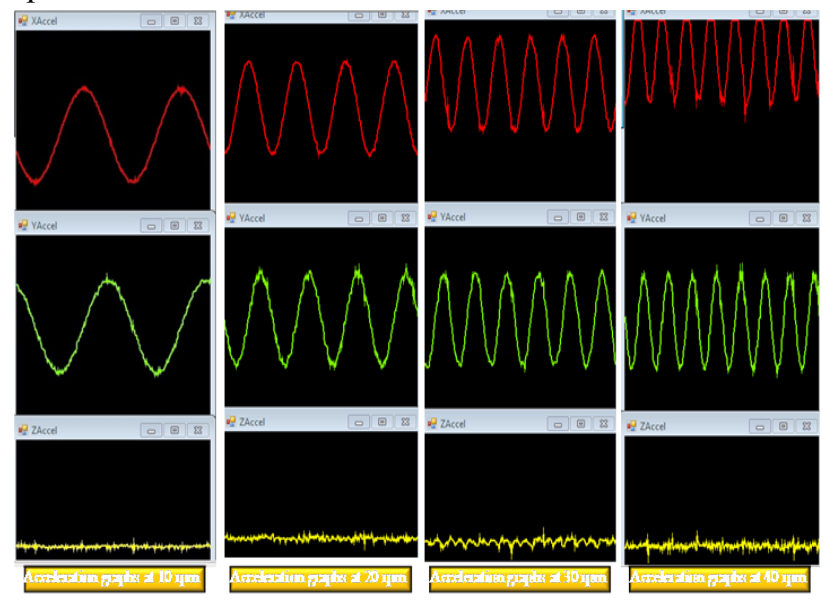

Figure 2. Vibration acceleration graphs in $\mathrm{X}, \mathrm{Y}$ and $\mathrm{Z}$ directions at different rotational speeds

\section{SOFTWARE DEVElOPMENT}

The general concept of the proposed software development is that the speed of a vehicle can be measured using an accelerometer based wireless sensor. The vibration acceleration data from the sensor are transferred wirelessly to a computer known as a server. The data are then processed in the server to convert to relevant vehicle speed data and transferred wirelessly to a desktop computer, known as client. The system is designed to continuously monitor the speed data displayed in a graphical User Interface (GUI). If the data value is greater than a pre-set speed limit, a report is generated with the details of the vehicle and its speed at that instantaneous time to be stored in the server. Therefore, the software system will have the capability of managing the data related to vehicle speed automatically and wirelessly. Fig. 3 presents the general architecture of the of the software development.

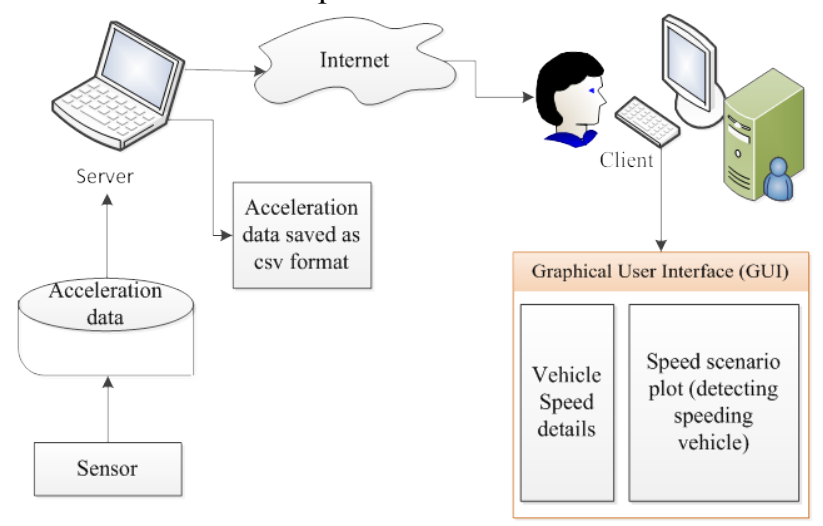

Figure 3. Software architecture

The software (SpeedManage) has been developed using Java ${ }^{\circledR}$ socket programming language. The vibration acceleration of the wheel is processed using a server socket application in the server computer. The server computes resultant vibration acceleration from the average vibration acceleration data and finally converts the data to speed format using the linear relationship established between vibration acceleration and the wheel speed. 
A connection has been established between the server and the client using internet to start communicating between them. A socket client algorithm has been used to create a GUI, which shows the vehicle speed plot with time. If the vehicle exceeds a particular speed limit set by the user on the client computer, the said speed of the car is considered as an over-speed on that road.

The over-speed is indicated on the GUI using a red coloured box. The speeds of the vehicle at different time are indicated using blue coloured boxes when the speeds of the car are below the speed limit. Amber coloured boxes in the GUI indicate that the speed of the vehicle, at that particular moment of time is equal to the maximum speed limit on the road. A horizontal red line is drawn on the GUI reflecting the maximum speed limit for the road. The client computer stores the information of the speeding vehicle such as Car number, road speed limit, speed of the car, time and date either as .csv (text) or pdf file formats

\section{Vehicle Speed Monitoring System}

\section{A. Scenario Design}

Two scenarios have been considered for a single vehicle running inside and outside the city. Different speed limits have been considered based on different roads. Fig. 4 displays the vehicle speed scenarios. The system is capable of setting the speed limit for both scenarios and proposed software interface can display the status of vehicle speed in both cases. The first scenario can simulate the vehicle speed measuring and monitoring situation inside a city/town, where the speed limit is assumed to be $40 \mathrm{~km} / \mathrm{hr}$ whereas the second scenario considers the situation in highway with a speed limit 60 $\mathrm{km} / \mathrm{hr}$. The speed limit can be set using a drop down menu from Speed Limit Settings dialog box in the client computer (Fig. 5).

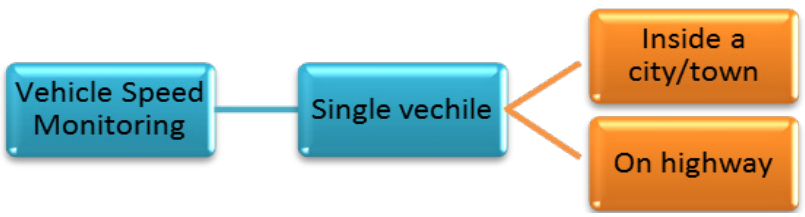

Figure 4. Vehicle speed scenarios for single vehicle

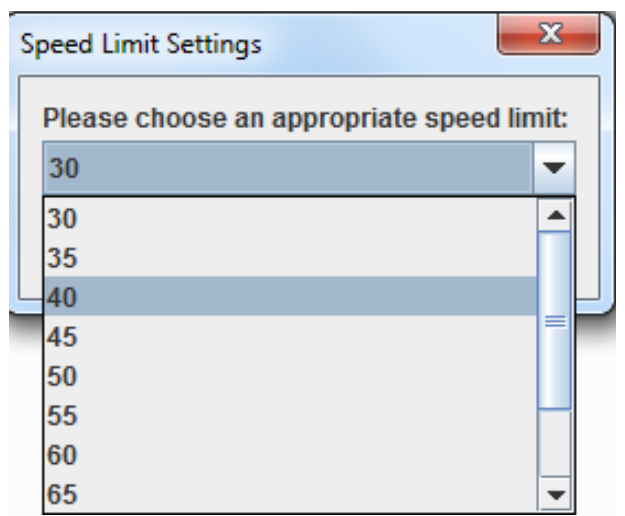

Figure 5. Selection of speed limit in the client computer for the vehicle running inside a city/town

\section{B. Simulation Results}

The vehicle speed details are displayed in the server computer. At the same time, the vehicle speed data with car number and other details are displayed in a server dialog box (Fig. 6). A DOS window is also generated in the client computer where all the speed data will be updated and displayed as a chart for monitoring purpose.

\begin{tabular}{|c|c|}
\hline Server & \begin{tabular}{|l|l|l}
$口$ & 口 & $x$ \\
\end{tabular} \\
\hline Car_number: & Car_1000 \\
\hline XAccel: & 2598 \\
\hline YAccel: & 1518 \\
\hline ZAccel: & 1800 \\
\hline Resultant_Acceleration: & 3506.27 \\
\hline RPM: & 15.21 \\
\hline Speed: & 30.42 \\
\hline
\end{tabular}

Figure 6. Display of latest speed data with vehicle details in a server dialog box for the vehicle running inside a city/town

The SpeedManage generates a graphical user interface (GUI) in the client computer. Fig. 7 displays a snapshot of vehicle speed plot at any particular moment. The GUI shows a red line across the plot, which indicates the speed limit set for the particular road traffic condition. The speed plot generally displays the speeds in every 30 seconds. Each point in the plot displays the actual value of the speed. The plot highlights the speed situations with different colours. Red colour indicates that the vehicle is running at a higher speed than the pre-set limit on the road. On the other hand, a blue colour indicates that the vehicle is running below the speed limit. Vehicle speed equal to the speed limit is indicated by the amber colour. The speed details are also continuously updated on the left hand side of the GUI.

The system can automatically detect the speeding vehicle and generate a report both in csv (text) and pdf file formats and save in the client computer. Therefore, the report can be sent to the offender for imposing penalty. An example of a pdf report has been presented in Fig. 8.

In case of vehicle running outside a city/town, it is assumed that the maximum speed limit is $60 \mathrm{~km} / \mathrm{hr}$. The speed limit can be set using a drop down menu from Speed Limit Settings dialog box in the client computer. Fig. 9 displays the snapshot of vehicle speeds with respect to time outside the city. Again at any time the software automatically picks up the speeding situation, generates report and saves the report automatically.

The flexibility of the proposed vehicle speed monitoring system shows great advantages in terms of detecting speeding vehicle, speed monitoring, sending and saving the detailed records of the offending vehicle to a central database automatically and instantly without any involvement of human effort. Thus, the cost for monitoring vehicle speed and managing the associated data can be significantly reduced using this automated system. Therefore, the system can play a vital role in integrating all the activities related to vehicle speed monitoring in an intelligent and efficient way. 


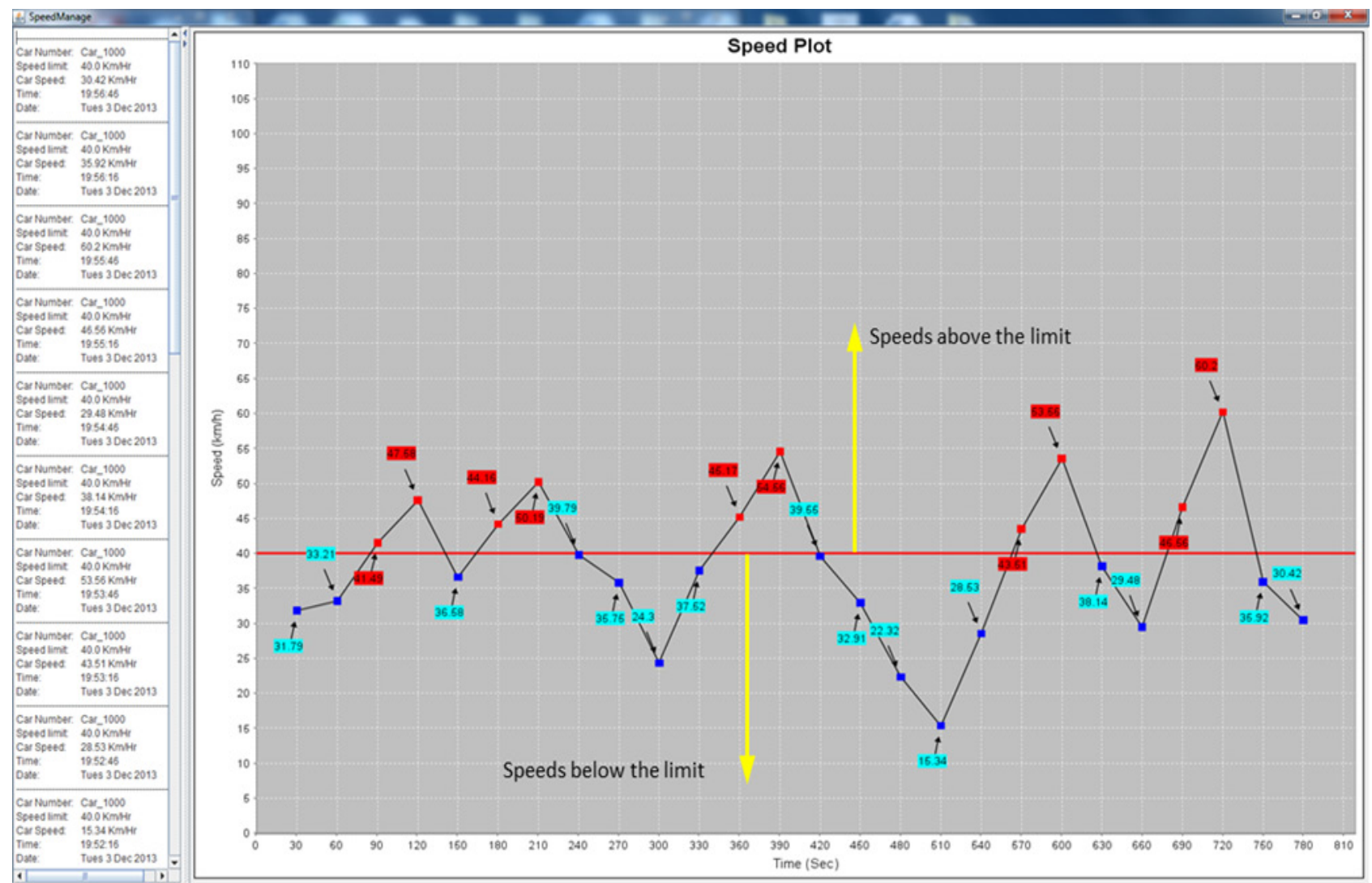

Figure 7. Display of vehicle speed plot on a GUI in the client computer for the vehicle running inside a city/town

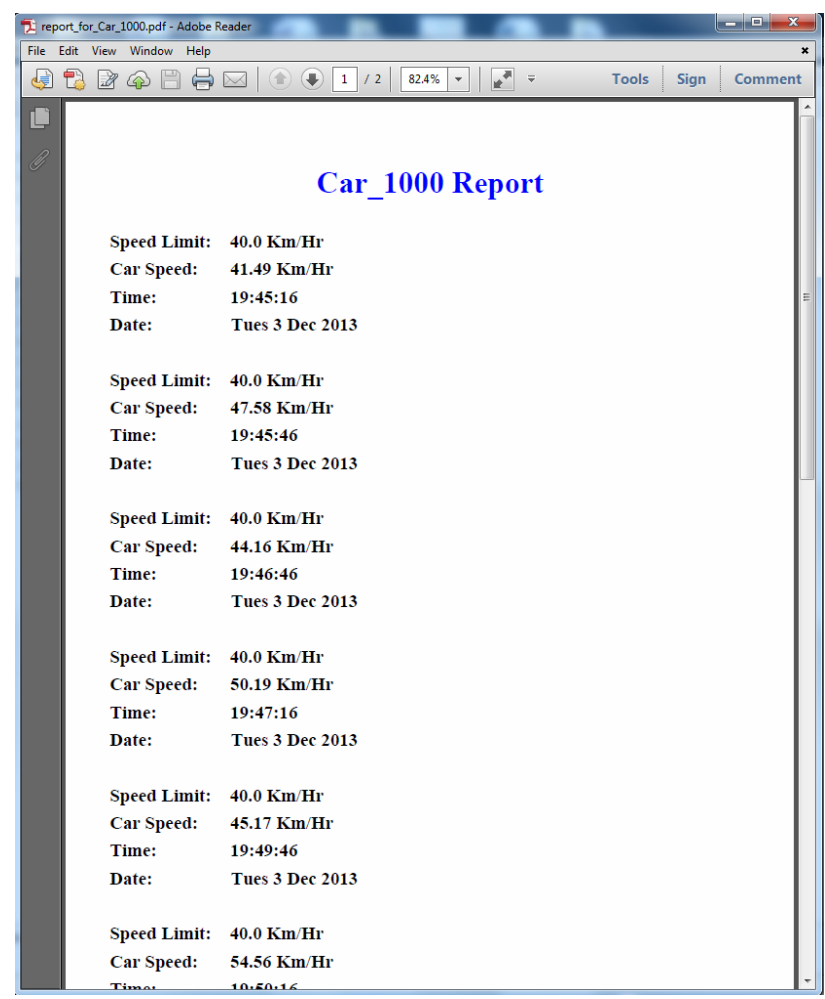

Figure 8. Report in pdf format for speeding vehicle inside a city/town

\section{CONCLUSIONS}

Software development, data acquisition, data analysis and management have been conducted for monitoring vehicle speed wirelessly in a simulated environment. A wireless sensor attached to a mechanical wheel can be used for measuring the wheel speed by sensing the wheel vibration acceleration where the wheel acts as a vehicle in the speed monitoring simulation.

An intelligent data management software interface, (SpeedManage) has been developed using Java ${ }^{\circledR}$ socket programming language that converts the wheel vibration acceleration data into speed format automatically. Additionally, the Java ${ }^{\circledR}$ interface transfers vehicle data wirelessly from a server computer to a client computer. The system is capable of detecting a speeding vehicle by comparing with a set speed limit, generating a report in the event of speeding and sending the report to the server database for storing. The system has been successfully tested with different speed limit scenarios for single vehicle. The GUI displays the data as red colour to identify the speeding vehicle inside the city with a speed limit set at $40 \mathrm{~km} / \mathrm{hr}$ and outside the city with a speed limit of $60 \mathrm{~km} / \mathrm{hr}$. Therefore, based on the performance of prototype system, it can be concluded that wireless sensorbased vehicle speed monitoring system has great potential for monitoring vehicle speed wirelessly.

\section{ACKNOWLEDGMENT}

The authors would like to acknowledge the support from the School of Mechanical and Manufacturing engineering, Dublin City University.

\section{REFERENCES}

[1] http://www.roadsafetymayo.ie/CausesofAccidents/

[2] C. Bachmann, B. Abdulhai, M.J. Roorda and B. Moshiri, "A comparative assessment of multi-sensor data fusion techniques for freeway traffic speed estimation using microsimulation modeling". Transp. Res. Part C, vol. 26, pp. 33-48, 2013. 


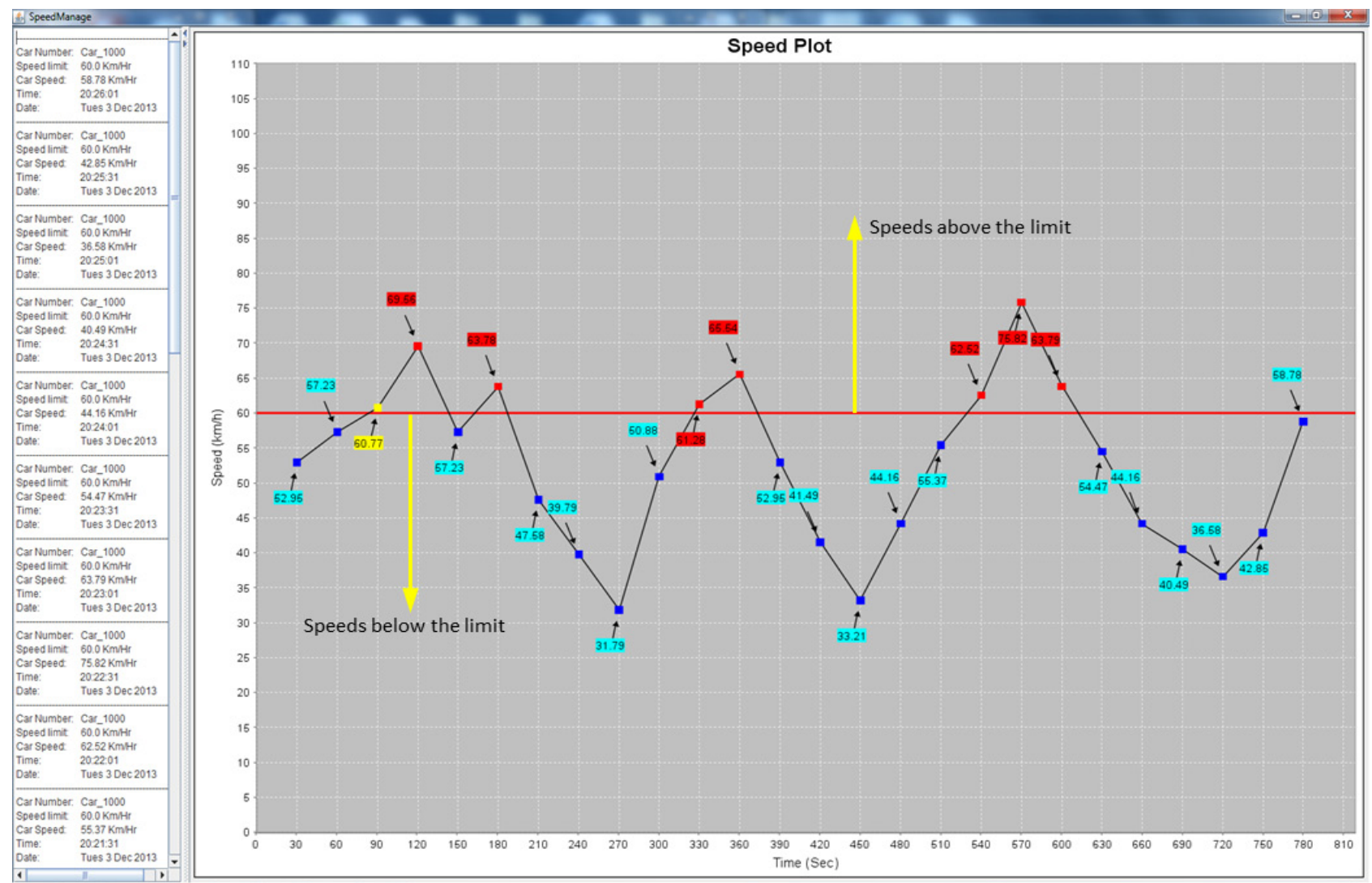

Figure 9. Display of vehicle speed plot on a GUI in the client computer for the vehicle running outside a city/town

[3] T. Matsuo, Y. Kaneko, and M. Matano, "Introduction of intelligent vehicle detection sensors," Proceedings IEEE/IEEJ/JSAI International Conference, Japan, pp. 709-713, 1999.

[4] W. Ma, D. Xing, A. McKee, R. Bajwa, C. Flores, B. Fuller, and P. Varaiya, "A wireless accelerometer-based automatic vehicle classification prototype system", IEEE Trans. Intel. Transp., vol. 15 , no.1, pp.104-111, 2014.

[5] M. Ahsan, "Development of wireless prototype vehicle speed monitoring system", MEng Thesis, submitted to Faculty of Engineering and Computing, Dublin City University, Ireland, 2014 .

[6] X. Chen, J. Zhang, S. Qian, and P. Xu, "Applied Research on Traffic Information Collection Based on Wireless Sensor Networks," International Conference on Future Electrical Power and Energy System, vol. 17, Part A, pp. 602-606, 2012.

[7] E. V. Roberts, Jr., "Traffic monitoring system and method," US Pat. No. 6384739B1, 2002.

[8] D. Douxchamps, B. Macq, and K. Chihara, "High Accuracy Traffic Monitoring using Road-Side Line-Scan Cameras," Proc. IEEE Intelligent Transportation Systems Conference (ITSC 06), pp. $875-878$, Sept. 2006

[9] J. Zhou, D. Gao, and D. Zhang, "Moving vehicle detection for automatic traffic monitoring," IEEE Trans. Veh. Technol., vol. 56 , no. 1, pp. 51-59, Jan 2007.

[10] B. Hull, V. Bychkovsky, Y. Zhang, K. Chen, M. Goraczko, A. Miu, E. Shih, H. Balakrishnan, and S. Madden, "CarTel: A Distributed Mobile Sensor Computing System," Proc. 4th Int Conf. Embedded Networked Sensor Systems (SenSys 06), pp. 125138, Nov. 2006

[11] S. Messelodi, C. M. Modena, M. Zanin, F. G. B. De Natale, F. Granelli, E. Betterle, and A. Guarise, "Intelligent Extended
Floating Car Data Collection," Expert Syst. Appl., vol. 36, no. 3, Part 1, pp. 4213-4227, 2009.

[12] X. Li, W. Shu, M. Li, H.-Y. Huang, P.-E. Luo, and M.-Y. Wu, "Performance Evaluation of Vehicle-Based Mobile Sensor Networks for Traffic Monitoring," IEEE T. Veh. Technol., vol. 58, no. 4, pp. 1647-1653, 2009.

[13] T. Nadeem, S. Dashtinezhad, C. Liao, and L. Iftode, "TrafficView: a scalable traffic monitoring system," Proc. IEEE International Conference on Mobile Data Management, pp. 13-26, 2004.

[14] S.K. Duvvuru, "Design and Deployment of Vehicular Traffic Monitoring Sensor Networks," Dissertation, M. Tech, Indian institute of technology, Bombay, 2008.

[15] S. Coleri, S.Y. Cheung, P. varaiya, "Sensor Networks for Monitoring Traffic," 42nd Annual ALLERTON Conference on Communication, Control, and Computing, pp. 883-892, September 2004.

[16] B. Karthikeyan, and M.Tamileniyan, "Dynamic Data update for Intelligent Speed Adaptation (ISA) System," Int. J. Comp. Appl. Tecnol., vol. 11, no.1, pp. 8-13, 2010.

[17] S. Luciani, Traffic monitoring system and method, US Pat. No. 6,505,114B2, 2003.

[18] D.V. Arnold, Jr. Dougall, J.B. Giles, B.C. Jarrett, B.R. Karlinsey, T.W., Waite, J.L. Systems and methods for monitoring speed. US Pat. No. 7426450B2, 2008.

[19] D.L. Jorgenson, M.G. Karlaftis, K.C. Sinha, "Vehicle speed considerations in traffic management: Development of a new speed monitoring program," J. Trans. Stat., vol. 3, no. 3, pp. 69$82,2000$.

[20] J.C. Herrera, D. B. Work, R. Herring, X. Ban, Q. Jacobson, and A M. Bayen, "Evaluation of traffic data obtained via GPS-enabled mobile phones," Trans. Res. Part C, vol. 18, no. 4, pp. 568-583, 2010

[21] Shimmer Research, www.shimmerresearch.com. 